\title{
User interestingness for pre-fetching in mobile environment
}

\begin{abstract}
In mobile environment, pre-fetching is used to prevent network congestion, delays and latency problems during disconnection. Lately, the pre-fetching strategies become more complicated in which to support new types of application especially in wireless environments. Due to this complication, new method is introduced where it requires data mining technique to improve the pre-fetching process. Previously, the pre-fetched data item is filtered using an data driven measurement for data interestingness and the data generated are based on the structure of a query pattern and quantified using statistical methods. The measurement is not good enough to solve the rule quality problems as in predicting next query in mobile environment. In this paper, a new measurement method is proposed to generate new criterion of pre-fetched data items before sending to mobile users. This method is improved by introducing user driven measurement for user interestingness where it will consider the subjectivity and the understandability of the users.
\end{abstract}

Keyword: Pre-fetching; Subjective measurement; User interestingness 